\title{
Emergent Failures and Cascades in Power Grids: A Statistical Physics Perspective
}

\author{
Tommaso Nesti, ${ }^{1}$ Alessandro Zocca, ${ }^{2}$ and Bert Zwart ${ }^{1}$ \\ ${ }^{1}$ CWI, Amsterdam 1098 XG, Netherlands \\ ${ }^{2}$ California Institute of Technology, Pasadena, California 91125, USA
}

(Received 25 July 2017; revised manuscript received 4 April 2018; published 21 June 2018)

\begin{abstract}
We model power grids transporting electricity generated by intermittent renewable sources as complex networks, where line failures can emerge indirectly by noisy power input at the nodes. By combining concepts from statistical physics and the physics of power flows and taking weather correlations into account, we rank line failures according to their likelihood and establish the most likely way such failures occur and propagate. Our insights are mathematically rigorous in a small-noise limit and are validated with data from the German transmission grid.
\end{abstract}

DOI: 10.1103/PhysRevLett.120.258301

Understanding cascading failures in complex networks is of great importance and has received a lot of attention in recent years [1-17]. Despite proposing different mechanisms for their evolution, a common feature is that cascades are triggered by some external event. This initial attack is chosen either (i) deliberately, to target the most vulnerable or crucial network component, or (ii) uniformly at random, to understand the average network reliability. This distinction led to the insight that complex networks are resilient to random attacks but vulnerable to targeted attacks $[7,18,19]$. However, both lead to the direct failure of the attacked network component.

In this Letter, we focus on networks in which edge failures occur in a fundamentally different manner. Specifically, we consider networks where fluctuations of the node inputs can trigger edge failures. The realization (which we call configuration) of the noise at the nodes not only is the cause of edge failures but can also impact the way they propagate in the network.

We present our results in the context of power grids that transport electricity generated by solar and wind parks. In power grids, line failures can arise when the network is driven from a stable state to a critically loaded state by external factors; intermittent power generation at the nodes causes random fluctuations in the line power flows, possibly triggering outages and cascading failures. Thus, line failures can emerge indirectly due to the interplay between noisy correlated (due to weather) power input at the nodes, the network structure, and power flow physics. This interplay is challenging to analyze, yet this problem is urgent as the penetration of renewable energy sources is increasing $[20,21]$.

We analyze this interplay using statistical physics and large-deviations theory. We consider a parsimonious static stochastic model similar to Ref. [22], introduce a scaling parameter $\varepsilon$ describing the magnitude of the noise, and consider the regime $\varepsilon \rightarrow 0$. In the limit, we can identify the most vulnerable lines and explicitly determine the most likely configuration of power inputs leading to failures and subsequent propagating failures. These results are validated using real data for the German transmission network. Previous works applying large-deviations techniques to problems in complex networks dynamics, such as epidemic extinction and biophysical networks, include Refs. [23,24].

We model a transmission network by a connected graph $G$ with $n$ nodes representing the buses and $m$ directed edges modeling transmission lines. The nominal values of net power injections at the nodes are given by $\boldsymbol{\mu}=\left\{\mu_{i}\right\}_{i=1, \ldots, n}$. We model the stochastic fluctuation of the power injections around $\boldsymbol{\mu}$, due to variability in renewable generation, by means of the random vector $\mathbf{p}=\left\{p_{i}\right\}_{i=1, \ldots, n}$, which is assumed to follow a multivariate Gaussian distribution with density

$$
\varphi(\mathbf{x})=\frac{\exp \left[-\frac{1}{2}(\mathbf{x}-\boldsymbol{\mu})^{T}\left(\varepsilon \boldsymbol{\Sigma}_{p}\right)^{-1}(\mathbf{x}-\boldsymbol{\mu})\right]}{(2 \pi)^{n / 2} \operatorname{det}\left(\varepsilon \boldsymbol{\Sigma}_{p}\right)^{1 / 2}},
$$

with $\varepsilon \boldsymbol{\Sigma}_{p} \in \mathbb{R}^{n \times n}$ being the covariance matrix of $\mathbf{p}$. In our theoretical analysis, we assume that $\boldsymbol{\Sigma}_{p}$ is known and let $\varepsilon \rightarrow 0$.

The Gaussian assumption is debatable, for both solar and wind. While consistent with atmospheric physics [25] and recent wind park statistics [26,27], different models are preferred for different timescales [28-31]. An extension of our framework to the dynamic model in Ref. [31] looks promising (using the Freidlin-Wentzell theory as in Ref. [32]). For a static non-Gaussian extension, see [33].

Assuming the vector $\boldsymbol{\mu}$ has zero sum and using the dc approximation [20], the line power flows $\mathbf{f}=\left\{f_{i}\right\}_{i=1, \ldots, m}$ are given by

$$
\mathbf{f}=\mathbf{V p},
$$

where $\mathbf{V}$ is an $m \times n$ matrix encoding the grid topology and parameters (i.e., line susceptances). The dc approximation 


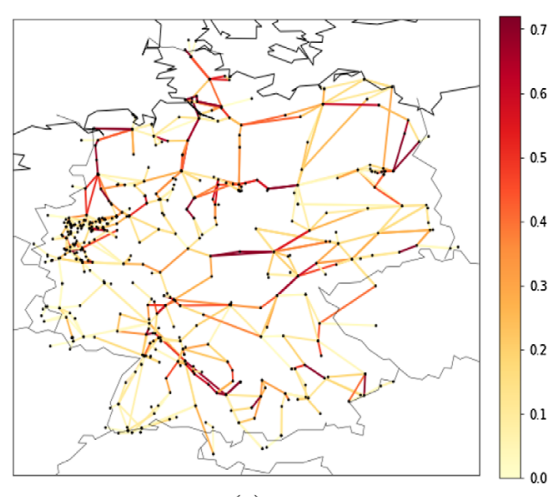

(a)

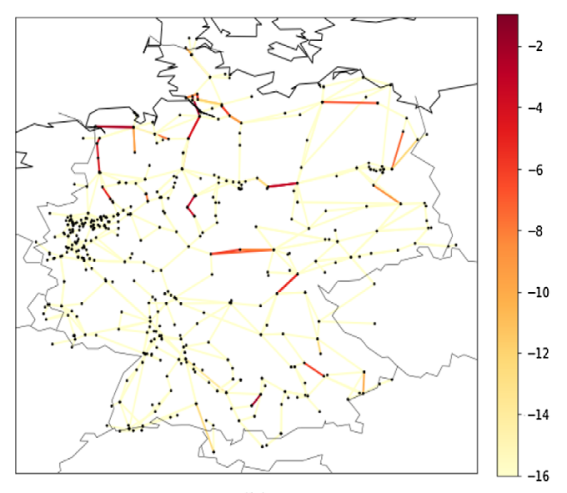

(b)

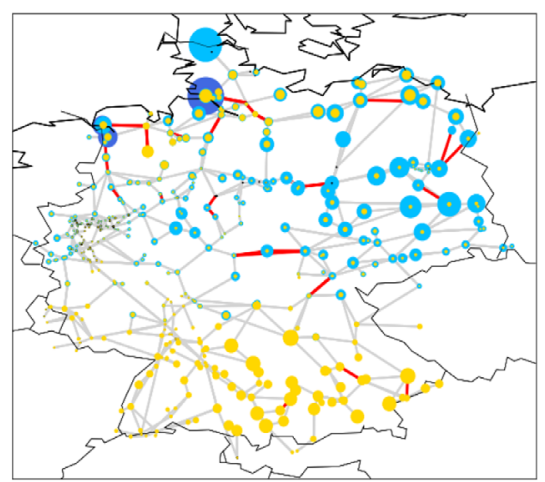

(c)

FIG. 1. (a) Nominal line flows $\left|\nu_{\ell}\right|$ at 11 am. (b) True overload probabilities $\log _{10} \mathbb{P}\left(\left|f_{\ell}\right| \geq 1\right)$ at 11 am. (c) Top 5\% of most likely lines to fail (red) at $11 \mathrm{am}$, according to (3), and nominal injections from renewable sources.

is commonly used in transmission system analysis [52-55]. More realistic nonlinear models based on ac power flows [56] may be analyzed leveraging the contraction principle [57].

The total net power injected in the network $\sum_{i=1}^{n} p_{i}$ is nonzero, as $\mathbf{p}$ is random. Automated affine response and redispatch mechanisms take care of this issue in power grids. Mathematically, this corresponds to a "distributed slack" in our model: The total power injection mismatch is distributed uniformly among all nodes (the matrix $\mathbf{V}$ accounts for this; see [33]).

In view of Eqs. (1) and (2), the line power flows $\mathbf{f}$ also follow a multivariate Gaussian distribution with mean $\boldsymbol{\nu}$ and covariance matrix $\varepsilon \boldsymbol{\Sigma}_{f}$. The vector $\boldsymbol{\nu}=\mathbf{V} \boldsymbol{\mu} \in \mathbb{R}^{m}$ describes the nominal line flows, while the covariance matrix $\varepsilon \boldsymbol{\Sigma}_{f}=\varepsilon \mathbf{V} \boldsymbol{\Sigma}_{p} \mathbf{V}^{T}$ describes the correlations between line flow fluctuations, taking into account both the correlations of the power injections (encoded by $\boldsymbol{\Sigma}_{p}$ ) and correlations created by the network topology due to power flow physics (Kirchhoff's laws) via V.

A line overloads if the absolute amount of power flowing in it exceeds a given line threshold. We assume that such overloads immediately lead to the outage of the corresponding line, to which we will henceforth refer simply as a line failure. The rationale behind this assumption is that there are security relays on high-voltage transmission lines performing an emergency shutdown as soon as the current exceeds a dangerous level. Without such mechanisms, lines may overheat, sag, and eventually trip.

We can express the line flows in units of the line threshold by incorporating the latter in the definition of $\mathbf{V}$ [33], so that $\mathbf{f}$ is the vector of normalized line power flows and the failure of line $\ell$ corresponds to $\left|f_{\ell}\right| \geq 1$. We let the power grid operate on average safely by assuming that $\max _{\ell=1, \ldots, m}\left|\nu_{\ell}\right|<1$, so that only large fluctuations of line flows lead to failures.

We are most interested in scenarios where power grids are highly stressed, meaning that the nominal power injections $\left\{\mu_{i}\right\}_{i=1, \ldots, n}$ are such that the corresponding nominal line power flows $\left\{\nu_{\ell}\right\}_{\ell=1, \ldots, m}$ are close to their thresholds. Such a stress could be caused by very high wind generation [58].

An illustrative scenario is reported in Fig. 1(a), which depicts a snapshot of nominal line flows on the SciGRID German network [59]. SciGRID is a detailed model of the actual German transmission network with $n=585$ buses and $m=852$ lines that we use as a main illustration. The data set includes load and generation time series, line limits, grid topology, and generation costs. In our case study, we obtain $\boldsymbol{\mu}$ by solving an optimal power flow (OPF) problem [60] based on realistic data for wind and solar generation, and we estimate $\varepsilon \boldsymbol{\Sigma}_{p}$ using autoregressive moving average models; for details, see Supplemental Material [33], which also describes a setting covering conventional controllable power plants.

We now turn to the analysis of emergent failures and their propagation using large-deviations theory [61]. We begin by deriving the exponential decay of probabilities of single line failure events $\left|f_{\ell}\right| \geq 1$ for $\ell=1, \ldots, m$. As line power flows are Gaussian, we obtain (see Example 3.1 in Ref. [61]) that

$$
I_{\ell}=-\lim _{\varepsilon \rightarrow 0} \varepsilon \log \mathbb{P}_{\varepsilon}\left(\left|f_{\ell}\right| \geq 1\right)=\frac{\left(1-\left|\nu_{\ell}\right|\right)^{2}}{2 \sigma_{\ell}^{2}}
$$

where $\sigma_{\ell}^{2}=\left(\boldsymbol{\Sigma}_{f}\right)_{\ell \ell}$. We call $I_{\ell}$ the decay rate of the failure probability of line $\ell$. Thus, for small $\varepsilon$, we approximate the probability of the emergent failure of line $\ell$ as

$$
\mathbb{P}\left(\left|f_{\ell}\right| \geq 1\right) \approx \exp \left(-I_{\ell} / \varepsilon\right)=\exp \left(-\frac{\left(1-\left|\nu_{\ell}\right|\right)^{2}}{2 \varepsilon \sigma_{\ell}^{2}}\right)
$$

and that of the first emergent failure as

$$
\mathbb{P}\left(\max _{\ell}\left|f_{\ell}\right| \geq 1\right) \approx \exp \left(-\min _{\ell} I_{\ell} / \varepsilon\right) .
$$

These approximations for failure probabilities may not be sharp, in general, even when $\varepsilon$ is small, since all terms that 


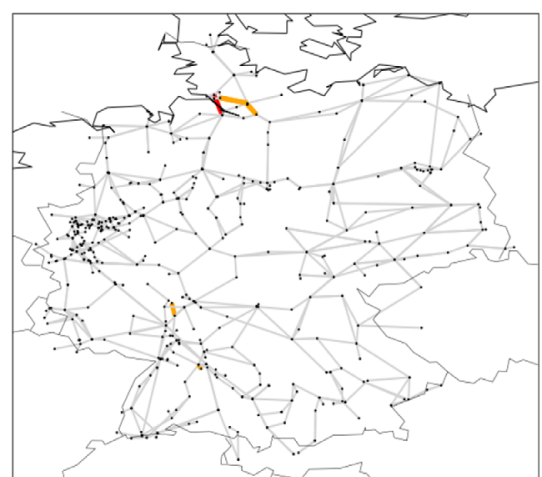

(a)

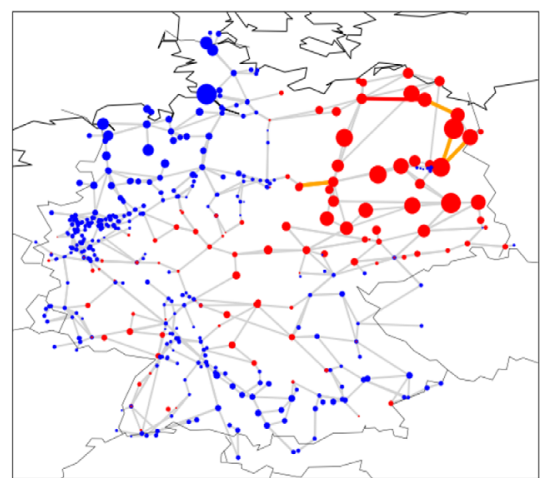

(b)

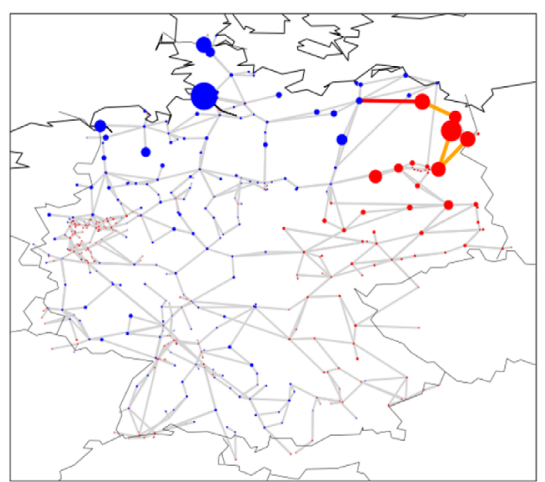

(c)

FIG. 2. (a) After the emergent failure of line 27 (red), six additional lines (orange) fail, 4 pm. (b) Most likely power injection $\mathbf{p}^{(\ell)}$ causing the isolated failure of line 720 (red) and subsequent failures (orange). The bus sizes reflect how much $\mathbf{p}^{(\ell)}$ deviates from $\boldsymbol{\mu}$ at 11 am (red, positive deviations; blue, negative). Left, with correlation in noise; right, without correlation in noise (setting to 0 all the offdiagonals of $\boldsymbol{\Sigma}_{p}$ ).

are decaying subexponentially in $1 / \varepsilon$ are ignored. Nevertheless, Eq. (4) is quite useful for ranking purposes, allowing us to explicitly identify the lines that are most likely to fail. To verify this empirically, we note that the expression in Eq. (4) depends only on the product $\varepsilon \sigma_{\ell}^{2}=\varepsilon\left(\mathbf{V} \boldsymbol{\Sigma}_{p} \mathbf{V}^{T}\right)_{\ell \ell}$ and thus, ultimately, only on the product $\varepsilon \boldsymbol{\Sigma}_{p}$, which in our case study we estimate directly from the SciGRID data; see [33].

Figure 1(b) shows the heat map for the exact line failure probabilities $\mathbb{P}\left(\left|f_{\ell}\right| \geq 1\right)$, for the same day and hour as in Fig. 1(a): It is clear that a larger $\left|\nu_{\ell}\right|$ does not necessarily imply a higher chance of failure. Figure 1(c) depicts the 5\% most likely lines to fail, ranked according to $I_{\ell}$. The ranking based on the large-deviations approximation successfully recovers the most likely lines to fail and, in fact, yields the same ordering as the one based on exact probabilities [33], thus providing an accurate indicator of system vulnerabilities.

Figure 1(c) also illustrates the nominal renewable generation mix: The buses housing stochastic power injections have different colors [blue (light blue) for wind offshore (onshore), yellow for solar] and sizes proportional to the absolute values of the corresponding nominal injections. Many vulnerable lines are located where the most renewable energy production occurs. However, the interplay between network topology, power flows physics, and correlation in power injections caused by weather fluctuations results in a spread-out arrangement of vulnerable lines, which is hard to infer by looking at nominal values only.

We proceed with an analysis of how emergent failures occur, using again large-deviations theory. In particular, we provide an explicit estimate of the most likely power injection that caused a specific emergent failure. To this end, we fix a line $\ell$ and consider the conditional distribution of $\mathbf{p}$, given $\left|f_{\ell}\right| \geq 1$. The mean of this distribution greatly simplifies as $\varepsilon \rightarrow 0$ to

$$
\mathbf{p}^{(\ell)}=\underset{\mathbf{p} \in \mathbb{R}^{n}: \hat{\mathbf{e}}_{\ell}^{T} \mathbf{V} \mathbf{p} \mid \geq 1}{\operatorname{arginf}} \frac{1}{2}(\mathbf{p}-\boldsymbol{\mu})^{T} \boldsymbol{\Sigma}_{p}^{-1}(\mathbf{p}-\boldsymbol{\mu}) .
$$

If $\nu_{\ell} \neq 0$, the solution is unique and reads

$$
\mathbf{p}^{(\ell)}=\boldsymbol{\mu}+\frac{\left[\operatorname{sgn}\left(\nu_{\ell}\right)-\nu_{\ell}\right]}{\sigma_{\ell}^{2}} \boldsymbol{\Sigma}_{p} \mathbf{V}^{T} \hat{\mathbf{e}}_{\ell}
$$

where $\operatorname{sgn}(a)=1$ if $a \geq 0$ and -1 otherwise and $\hat{\mathbf{e}}_{\ell} \in \mathbb{R}^{m}$ is the $\ell$ th unit vector. As $\varepsilon \rightarrow 0$, the conditional variance of p given $\left|f_{\ell}\right| \geq 1$ decreases to 0 exponentially fast in $1 / \varepsilon$, yielding that the conditional distribution of $\mathbf{p}$ given $\left|f_{\ell}\right| \geq 1$ gets sharply concentrated around $\mathbf{p}^{(\ell)}$ [33].

We interpret $\mathbf{p}^{(\ell)}$ as the most likely power injection profile, conditional on the failure of line $\ell$. The corresponding line power flow profile $\mathbf{f}^{(\ell)}=\mathbf{V} \mathbf{p}^{(\ell)}$ is

$$
f_{k}^{(\ell)}=\nu_{k}+\frac{\left[\operatorname{sgn}\left(\nu_{\ell}\right)-\nu_{\ell}\right]}{\sigma_{\ell}^{2}} \operatorname{Cov}\left(f_{\ell}, f_{k}\right), \quad \forall k \neq \ell .
$$

As such, our framework provides more explicit information than the approach in Ref. [62], which approximates the most likely way events happen using the mode, without leveraging large deviations. In our validation experiments, we found that the error between $\mathbf{p}^{(\ell)}$ and $\mathbb{E}\left[\mathbf{p}|| f_{\ell} \mid \geq 1\right]$ is typically less than $1 \%$ of the nominal values [33]. A numerical illustration is given in Fig. 2(b).

A key finding is that an emergent line failure does not occur due to large fluctuations only in neighboring nodes but as a cumulative effect of small unusual fluctuations in the entire network "summed up" by power flow physics and correlations in renewable energy. Such an emergent failure requires every line flow to be driven to an unusual state $f_{k}^{(\ell)}$, which deviates from the nominal value $\nu_{k}$ by an amount proportional to the covariance $\operatorname{Cov}\left(f_{\ell}, f_{k}\right)$, in view of Eq. (7). 
We continue by investigating the propagation of failures, combining our results describing the most likely power injections configuration leading to the first failure and the power flow redistribution in the network afterwards. To this end, we first differentiate between different types of line failures, by assessing whether the most likely way for failure of line $\ell$ to occur is as (i) an isolated failure, if $\left|f_{k}^{(\ell)}\right|<1$ for all line $k \neq \ell$, or (ii) a joint failure, if there exists some other line $k \neq \ell$ such that $\left|f_{k}^{(\ell)}\right| \geq 1$.

Any type of line failure(s) cause(s) a global redistribution of the line power flows according to Kirchhoff's laws, which could trigger further outages and cascades. In our setting, the power injections configuration $\mathbf{p}^{(\ell)}$ redistributes across an altered network $\tilde{G}^{(\ell)}$ (a subgraph of the original graph $G$ ) in which line $\ell$ (and possible other lines, in case of a joint failure) has been removed, increasing stress on the remaining lines. The way this redistribution happens on $\tilde{G}^{(\ell)}$ is governed by power flow physics, and we assume that it occurs instantaneously. Extending this to dynamic models $[63,64]$ is a natural future topic, as transient oscillatory effects may aggravate the impact of line failures.

The power flow redistribution amounts to computing a new matrix $\tilde{\mathbf{V}}$ linking the power injections and the new power flows, which can be constructed analogously to $\mathbf{V}$ [33]. The most likely power flow configuration on $\tilde{G}^{(\ell)}$ after redistribution is $\tilde{\mathbf{f}}^{(\ell)}=\tilde{\mathbf{V}} \mathbf{p}^{(\ell)}$.

In the special case of an isolated failure (say, of line $\ell$ ), it is enough to calculate the vector $\boldsymbol{\phi}^{(\ell)} \in \mathbb{R}^{m-1}$ of (normalized) redistribution coefficients, known as line outage distribution factors (LODF) [65]. The quantity $\phi_{j}^{(\ell)}$ takes values in $[-1,1]$, and $\left|\phi_{j}^{(\ell)}\right|$ represents the percentage of power flowing in line $\ell$ that is redirected to line $j$ after the failure of the former. The most likely power flow configuration on $\tilde{G}^{(\ell)}$ after redistribution then equals $\quad \tilde{\mathbf{f}}^{(\ell)}=\left\{f_{k}^{(\ell)}\right\}_{k \neq \ell}+f_{\ell}^{(\ell)} \boldsymbol{\phi}^{(\ell)}$, where $f_{\ell}^{(\ell)}= \pm 1$ depending on the way the power flow is most likely to exceed the threshold 1 . The power flow configuration $\tilde{\mathbf{f}}^{(\ell)}$ can be efficiently used to determine which lines subsequently fail, by checking for which $k$ we have $\left|\tilde{f}_{k}^{(\ell)}\right| \geq 1$; see [33].

There is much evidence that failures propagate nonlocally in power grids [66-70]. To analyze this in our framework, we first consider a ring network with $\boldsymbol{\mu}=0$ and $\boldsymbol{\Sigma}_{p}=I$. In this network, there are two paths along which power can flow between any two nodes, using the convention that a positive flow corresponds to a counterclockwise direction. If line $\ell$ fails, the power originally flowing on line $\ell$ must now flow on the remaining path in the opposite direction. To make this rigorous, we show in Ref. [33] that $\phi_{k}^{(\ell)}=-1$ for every $k \neq \ell$. As power flows must sum to zero by Kirchhoff's law, neighboring lines tend to have positively correlated power flows, while flows on distant lines exhibit negative correlations. Hence, the power injections that make the power flows in line $\ell$ exceed
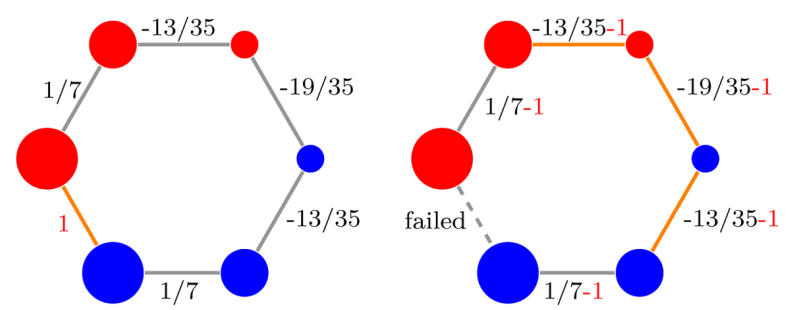

FIG. 3. Left: Most likely power injections $\mathbf{p}^{(\ell)}$ leading to the failure of line $\ell$ (orange), visualized using the color and size of the nodes (red, positive deviations; blue, negative), together with power flows $f_{k}^{(\ell)}$. Right: Situation after the power flow redistribution with three subsequent failures and the values $\tilde{f}_{k}^{(\ell)}=f_{k}^{(\ell)}-1, k \neq \ell$.

the line threshold (say, by becoming larger than 1) also make the power flows in the antipodal half of the network negative. These will go beyond the line threshold -1 after the power flow redistributes; cf. Fig. 3.

In the SciGRID example, Fig. 2(a) shows how the emergent isolated failure of line $\ell=27$ causes the failure of six more lines $k_{1}, \ldots, k_{6}$, two of which are far way from the original failure. For validation purposes, we found numerically that $\mathbb{P}\left(\right.$ line $k_{j}$ fails $\left.\forall j=1, \ldots, 6|| f_{27} \mid \geq 1\right) \geq$ 0.9987. Conversely, the failure of line 27 under the nominal power injection profile leads to only two subsequent failures. The nontypical input caused other lines to be more loaded than expected, and these lines get more vulnerable as the cascades progresses, resulting in more subsequent failures.

To validate this insight, we have looked at the first two stages of emergent cascading failures for several IEEE test networks and compare them with those of classical cascading failures, obtained using nominal power injection values rather than the most likely ones and deterministic removal of the initial failing line; see [33] for a precise description of the experiment. As before, emergent cascades tend to lead to a higher number of subsequent failures in each stage.

A nondiagonal noise matrix $\boldsymbol{\Sigma}_{p}$ exacerbates these effects. Experiments [see Fig. 2(b)] with our SciGRID case study suggest that, if there is a correlation in noise, for example, due to fluctuations in weather patterns, the number of subsequent failures can become higher. Furthermore, it is easier for a failure to be triggered by many small disturbances across the network, compared to the case where these correlations are not taken into account. In the latter case, we see a more local effect with relatively larger disturbances.

In conclusion, we illustrated the potential of concepts from statistical physics and large-deviations theory to analyze emergent failures and their propagation in complex networks. Exogenous noise disturbances at the nodes, potentially amplified by correlations, push a complex network into a critical state in which edge failure may emerge. Large-deviations theory provides a tool to rank such failures according to their likelihood and predicts how such failures most likely occur and propagate. When an 
emergent edge failure occurs, its impact on the network can be more significant than a purely exogenous failure, possibly resulting in cascades that propagate quicker than in a classical vulnerability analysis.

The accuracy of the small noise limit has been validated in our case study, making the case for applying large-deviations techniques to more realistic models. In Ref. [33], we propose a promising economic application of our approach, showing how our framework can shed light on the trade-off between network reliability and societal costs.

We thank the referees for many useful comments, in particular, for suggesting SciGRID. NWO Vici 639.033.413 and NWO Rubicon 680.50.1529 grants provided financial support. A. Z. acknowledges the support of Resnick Sustainability Institute at Caltech.

[1] R. Albert, I. Albert, and G. L. Nakarado, Phys. Rev. E 69, 025103 (2004).

[2] R. Albert and A.-L. Barabási, Rev. Mod. Phys. 74, 47 (2002).

[3] R. Albert, H. Jeong, and A.-L. Barabási, Nature (London) 406, 378 (2000).

[4] P. Crucitti, V. Latora, and M. Marchiori, Phys. Rev. E 69, 045104 (2004).

[5] P. Crucitti, V. Latora, M. Marchiori, and A. Rapisarda, Physica A 320, 622 (2003).

[6] B. Mirzasoleiman, M. Babaei, M. Jalili, and M. A. Safari, Phys. Rev. E 84, 046114 (2011).

[7] A. E. Motter and Y.-C. Lai, Phys. Rev. E 66, 065102 (2002).

[8] A. E. Motter, Phys. Rev. Lett. 93, 098701 (2004).

[9] D. Heide, M. Schäfer, and M. Greiner, Phys. Rev. E 77, 056103 (2008).

[10] R. Kinney, P. Crucitti, R. Albert, and V. Latora, Eur. Phys. J. B 46, 101 (2005).

[11] B. Schäfer, C. Beck, K. Aihara, D. Witthaut, and M. Timme, Nat. Energy 3, 119 (2018).

[12] S. Sun, Z. Liu, Z. Chen, and Z. Yuan, Physica A 373, 851 (2007).

[13] Y. Yang, T. Nishikawa, and A. E. Motter, Science 358, eaan3184 (2017).

[14] D. Watts, Proc. Natl. Acad. Sci. U.S.A. 99, 5766 (2002).

[15] D. Witthaut and M. Timme, Phys. Rev. E 92, 032809 (2015).

[16] D. Witthaut, M. Rohden, X. Zhang, S. Hallerberg, and M. Timme, Phys. Rev. Lett. 116, 138701 (2016).

[17] D. Witthaut and M. Timme, Eur. Phys. J. B 86, 377 (2013).

[18] R. Cohen, K. Erez, D. ben-Avraham, and S. Havlin, Phys. Rev. Lett. 85, 4626 (2000).

[19] R. Cohen, K. Erez, D. ben-Avraham, and S. Havlin, Phys. Rev. Lett. 86, 3682 (2001).

[20] D. Bienstock, Electrical Transmission System Cascades and Vulnerability (SIAM, Philadelphia, 2015), Chap. 4.

[21] I. Dobson, B. Carreras, V. Lynch, and D. Newman, Chaos 17, 026103 (2007).

[22] Z. Wang, A. Scaglione, and R. Thomas, in Proceedings of the 2012 45th Hawaii International Conference on System
Sciences (IEEE, New York, 2012), pp. 2115-2124, https:// doi.org/10.1109/HICSS.2012.63.

[23] D. K. Wells, W. L. Kath, and A. E. Motter, Phys. Rev. X 5, 031036 (2015).

[24] J. Hindes and I. B. Schwartz, Phys. Rev. Lett. 117, 028302 (2016).

[25] D. Bienstock, M. Chertkov, and S. Harnett, SIAM Rev. 56, 461 (2014).

[26] S. Kolumban, S. Kapodistria, and N. Nooraee, arXiv:1707.06497.

[27] J. Berg, A. Natarajan, J. Mann, and E. G. Patton, Wind Energ. 19, 1975 (2016).

[28] A. S. Brouwer, M. van den Broek, A. Seebregts, and A. Faaij, Renewable Sustainable Energy Rev. 33, 443 (2014).

[29] D. Schlachtberger, S. Becker, S. Schramm, and M. Greiner, Renew. Sustain. Energ. Rev. 125, 336 (2016).

[30] Y. Peings and G. Magnusdottir, Environ. Res. Lett. 9, 034018 (2014).

[31] P. Milan, M. Wächter, and J. Peinke, Phys. Rev. Lett. 110, 138701 (2013).

[32] T. Nesti, J. Nair, and B. Zwart, arXiv:1606.02986.

[33] See Supplemental Material at http://link.aps.org/ supplemental/10.1103/PhysRevLett.120.258301 for all the details about the power flow model, the large-deviations principle for failures, and the numerics for the German network, which includes Refs. [34-51].

[34] H. Cetinay, F. Kuipers, and P. Van Mieghem, IEEE Syst. J. 1 (2016).

[35] M. Schaub, J. Lehmann, S. Yaliraki, and M. Barahona, Network Sci. 2, 66 (2014).

[36] S. Soltan, D. Mazauric, and G. Zussman, IEEE Trans. Control Network Syst. 4, 288 (2017).

[37] R. Bapat, Ramanujan Math. Soc. Lect. Notes Ser. 7, 63 (2008).

[38] T. Brown, J. Hörsch, and D. Schlachtberger, J. Open Res. Software 6, 4 (2018).

[39] T. Brown, https://pypsa.org/examples/scigrid-lopf-then-pf .html.

[40] T. Brown, https://pypsa.org/examples/add_load_gen_ trafos_to_scigrid.html.

[41] OpenStreetMap contributors, Planet dump retrieved from https://planet.osm.org, https://www.openstreetmap.org.

[42] M. Milligan, M. Schwartz, and Y. Wan, National Renewable Energy Laboratory, Report No. NREL/CP-500-33956, 2003, https://www.nrel.gov/docs/fy03osti/33956.pdf.

[43] J. Antonanzas, N. Osorio, R. Escobar, R. Urraca, F. M. de Pison, and F. Antonanzas-Torres, Solar Energy 136, 78 (2016).

[44] R. Huang, T. Huang, R. Gadh, and N. Li, in Proceedings of the 2012 IEEE Third International Conference on Smart Grid Communications (SmartGridComm) (IEEE, New York, 2012), pp. 528-533.

[45] J. Zhang, B.-M. Hodge, and A. Florita, in Proceedings of the ASME 2013 7th International Conference on Energy Sustainability (IEEE, 2013), p. V001T16A003.

[46] B. M. Hodge and M. Milligan, in Proceedings of the 2011 IEEE Power and Energy Society General Meeting (IEEE, New York, 2011).

[47] F. Schweppe, M. Caramanis, R. Tabors, and R. Bohn, Spot Pricing of Electricity (Springer, New York, 1988). 
[48] P. Hines, I. Dobson, E. Cotilla-Sanchez, and M. Eppstein, in Proceedings of the 2013 46th Hawaii International Conference on System Sciences (IEEE, New York, 2013), pp. 2141-2150.

[49] P. Hines, I. Dobson, and P. Rezaei, IEEE Trans. Power Syst. 32, 1 (2016).

[50] J. Qi, K. Sun, and S. Mei, IEEE Trans. Power Syst. 30, 804 (2015).

[51] Y. Yang, T. Nishikawa, and A. E. Motter, Phys. Rev. Lett. 118, 048301 (2017).

[52] K. Purchala, L. Meeus, D. Van Dommelen, and R. Belmans, in Proceedings of the IEEE Power Engineering Society General Meeting (IEEE, New York, 2005), pp. 2457-2462, http://dx.doi.org/10.1109/PES.2005.1489581.

[53] B. Stott, J. Jardim, and O. Alsac, IEEE Trans. Power Syst. 24, 1290 (2009).

[54] L. Powell, Power System Load Flow Analysis (McGrawHill, New York, 2004).

[55] A. Wood, B. Wollenberg, and G. Sheble, Power Generation, Operation, and Control, 3rd ed. (Wiley, New York, 2014).

[56] D. Mehta, D. Molzahn, and K. Turitsyn, in Proceedings of the 2016 American Control Conference (ACC) (IEEE, New York, 2016), pp. 1753-1765, http://dx.doi.org/10.1109/ ACC.2016.7525170.

[57] A. Dembo and O. Zeitouni, Large Deviations Techniques and Applications, Stochastic Modelling and Applied Probability Vol. 38 (Springer, Berlin, 2010).
[58] T. Pesch, H.-J. Allelein, and J.-F. Hake, Eur. Phys. J. Spec. Top. 223, 2561 (2014).

[59] C. Matke, W. Medjroubi, and D. Kleinhans, SciGRID-An Open Source Reference Model for the European Transmission Network, https://www.scigrid.de/.

[60] M. Huneault and F. D. Galiana, IEEE Trans. Power Syst. 6, 762 (1991).

[61] H. Touchette, Phys. Rep. 478, 1 (2009).

[62] M. Chertkov, F. Pan, and M. Stepanov, IEEE Trans. Smart Grid 2, 162 (2011).

[63] I. Simonsen, L. Buzna, K. Peters, S. Bornholdt, and D. Helbing, Phys. Rev. Lett. 100, 218701 (2008).

[64] B. Schäfer, D. Witthaut, M. Timme, and V. Latora, arXiv:1707.08018.

[65] J. Guo, Y. Fu, Z. Li, and M. Shahidehpour, IEEE Trans. Power Syst. 24, 1633 (2009).

[66] D. Jung and S. Kettemann, Phys. Rev. E 94, 012307 (2016).

[67] S. Kettemann, Phys. Rev. E 94, 062311 (2016).

[68] D. Labavić, R. Suciu, H. Meyer-Ortmanns, and S. Kettemann, Eur. Phys. J. Spec. Top. 223, 2517 (2014).

[69] H. Ronellenfitsch, D. Manik, J. Horsch, T. Brown, and D. Witthaut, IEEE Trans. Power Syst. 32, 4060 (2017).

[70] D. Manik, M. Rohden, H. Ronellenfitsch, X. Zhang, S. Hallerberg, D. Witthaut, and M. Timme, Phys. Rev. E 95, 012319 (2017). 